\title{
STANDARDISED COMPUTER TESTS FOR ASSESSMENT OF CHILDREN MANUAL DEXTERITY
}

\author{
MAŁGORZATA KAMIENIARZ ${ }^{1}$, WANDA STRYŁA ${ }^{1}$, \\ PIOTR HAGLAUER ${ }^{2}$, GRZEGORZ KAMIENIARZ ${ }^{2}$ \\ ${ }^{1}$ Clinic of Rehabilitation, K. Marcinkowski University of Medical Sciences, \\ 28 Czerwca 1998, Poznań, Poland \\ ${ }^{2}$ Computational Physics Division, Institute of Physics, A. Mickiewicz University, \\ Umultowska 85, 61-614 Poznań, Poland
}

\begin{abstract}
The paper presents a computer program developed for the purpose of objectivisation of children hand dexterity. The program is composed of 6 subtests checking the control of the upper limb joints and the fine finger dexterity needed for mouse control. Measurements have been performed for a sample of 285 children aged from 7 to 15 . Clinical standards for particular age groups have been proposed and statistical significance of differences among the corresponding mean values has been shown. A strong correlation of the hand control and age of the subjects has been found.
\end{abstract}

\section{INTRODUCTION}

Progress in rehabilitation requires development of quantitative and objective methods for assessment of the patient abilities and disabilities as well as evaluation of the effects of treatment. Deficit in the motor functions in the developmental age leads to morphological deformations and is a substantial obstacle in acquiring competency in the activity of daily living, may also cause a disorder of manual skills and even secondary impairment in the posture [1]. Diagnosis of the locomotive system based on objective and comparable quantitative results ensures correct assessment and thus adequate choice of therapeutic methods. A correct diagnosis may determine the effects of treatment and is an inevitable element of monitoring the therapy.

The human hand is capable of complex and precise functions which can be divided into grasping abilities (measured by the strength and quality of grasp) and manual dexterity. The quality of grasp is the ability to adapt to a given object while the strength of grasp characterises the ability to withstand overload. The quality of grasp is determined by the range of movements in the joints, while its strength by the muscle power.

Manual dexterity is a manual skill requiring coordination of fme and gross movement based on a number of capacities developed through learning and experience. There are two main types of manual dexterity: fine and gross dexterity. Fine dexterity refers to the ability to manipulate objects using the distal part of the fingers. Gross manual dexterity or simply manual dexterity involves less refined and less precise movements of the hand and fingers.

In the last three decades measurement of muscle power of a hand in performing precise and strong grasps has been the subject of intense studies [2-9], however, mainly on population of adults. The results were found dependent on the upper limb position during measurements and accuracy of instruction. The standard position [7] was assumed that of the patient sitting down 
with the hand bent in the elbow at 90 degrees. Dynamometer measurements in this position led to establishment of clinical standards [7,8] for men and women aged 20 to 75. Measurement of individual muscle strength and joint range of motion gives some idea of what the patient may use his hands for. However, effective substitution patterns and patient motivation cause these tests to become inaccurate sources of information [10].

As far as evaluation of manual skills is concerned, literature provides many tests: the hand functional test proposed by Jebsen et al. [11, 12], Nine-Hole Peg Test and Fifty-Hole Peg Test [2], Motor Assessment Scale [13] based on checking skills in everyday activities [14, 15], tests checking functioning of upper extremities [16, 17], fingers and wrist [18, 19], functional tests [20] being certain modifications of the above. Among the recently proposed methods there are a new Sequential Occupational Dexterity Assessment [21] and an accelerometry-based test [22], In the latter, the electric signal from a transducer is proportional to the acceleration of the hand movement. The measurement is performed when the patient (a child with cerebral palsy) reaches out for raisins placed on the table and puts them into the mouth. As follows from the short descriptions of the above methods, neither of them has involved hand motor functions for computer control. Until recently, a computer has been used as an aid for dynamometric tests [8]. Reports on direct application of computer in diagnostics appeared only in recent years. For example dexterity of the elbow joint in post-stroke hemiparesis [23] was assessed by testing the ability to follow an object on the screen performing movements with the elbow. Unfortunately, no evaluation covers all aspects of hand function so that there is no consensus that any evaluation adequately measures the hand function. As no hand function evaluation is appropriate for all types of patients, clinicians should have a number of functional hand tests at their disposal [24].

The aim of the study reported in this paper was to work out computer aided tests checking the hand dexterity in children aged from 7 to 15 and to establish clinical standards for three age groups of healthy children taking into account performance of dominant and non-dominant hands. The tests are based on checking the control of the mouse and performance of a few specific tasks on the screen in time.

\section{TESTS DESCRIPTION}

Six tasks have been developed to check manipulation dexterity of the hand - called blocks (in Polish language - klocki), labyrinth (labirynt), ball (piłka), circle (koto), board (plansza) and centres (środki). The program is written in MS Visual Basic 5.0 and operates on PC platform in Windows 95 system. The user interface is worked out in Polish. Fig. 1 presents the starting menu of the application. The idea of the tests is based on the fact that the mouse control requires movements of all joints of the upper limb and on top of that, the isolated movement of the index finger, which is important for finger tip pinch. In the simplest version, used in the study, the mouse moves in the horizontal plane, which does not require rotations in the elbow. However, this restriction can be easily removed by using a table of variable inclination with respect to the horizontal plane. 
Fig. 1. The main menu of the program listing all the tasks
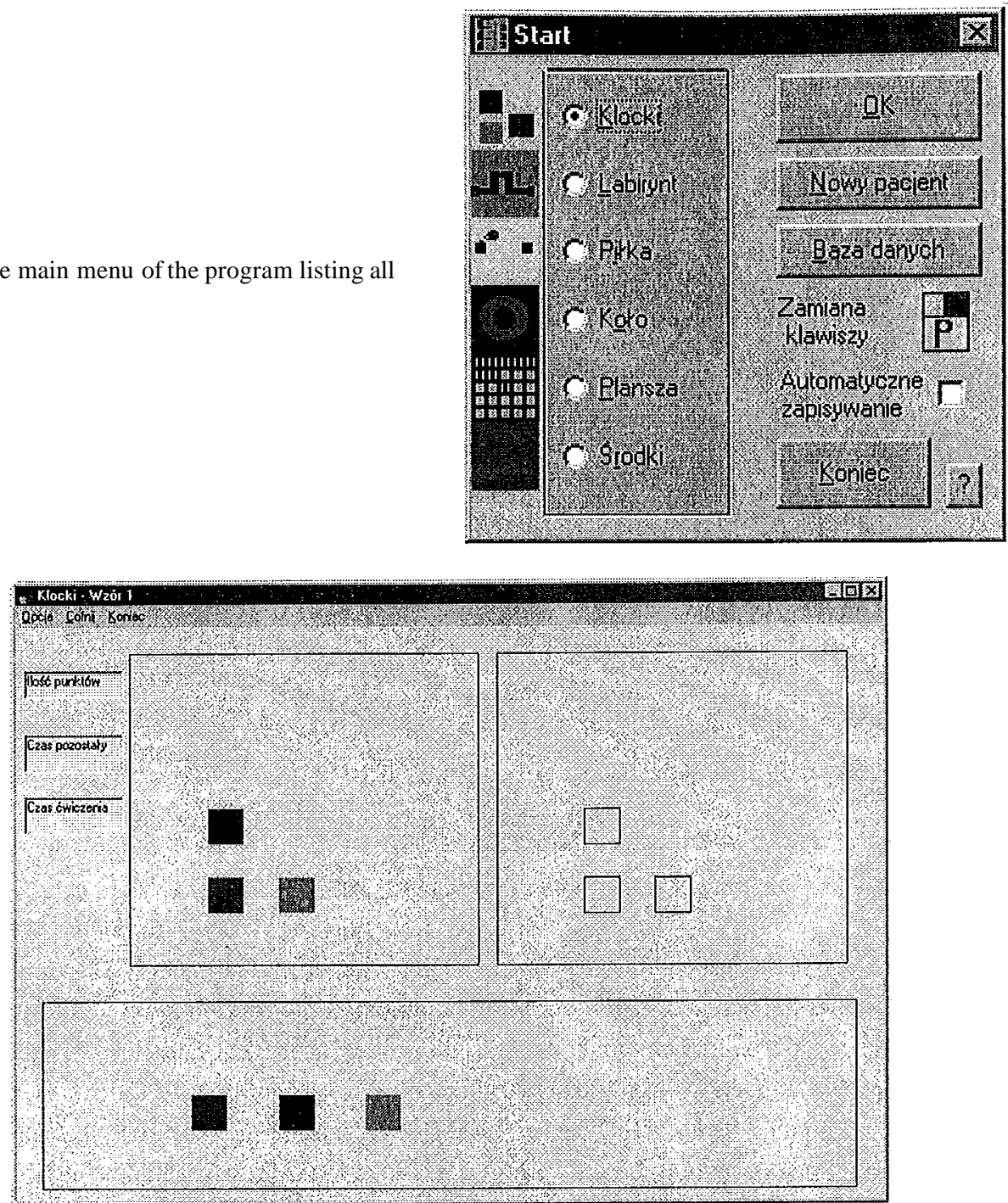

Fig. 2. The window of the subtest blocks (klocki)

The program-task blocks (Fig. 2) gives on the screen one window and three text fields which give the score, time left to complete the test and test duration. The person tested is asked to arrange colour blocks given in the bottom rectangle in the way shown in the left square. The blocks are arranged by driving the mouse cursor on the block, clicking the mouse and moving the block to a desired place. The block is released with another click of the left or right button, depending on the hand tested. The score depends on the number of correctly positioned blocks. The time of the tasks completion is limited to 120 seconds. 
To perform the task labyrinth, whose window is shown in Fig. 3, the person tested is asked to draw a broken line connecting the human figure with the tree. The task should be completed as quickly as possible and can be performed along a given path. The correctly covered path is shaded, whereas any deviation from the path is signalled by a sound and recorded as a mistake. The user can continue moving the cursor only having come back to the point at which the mistake was made.

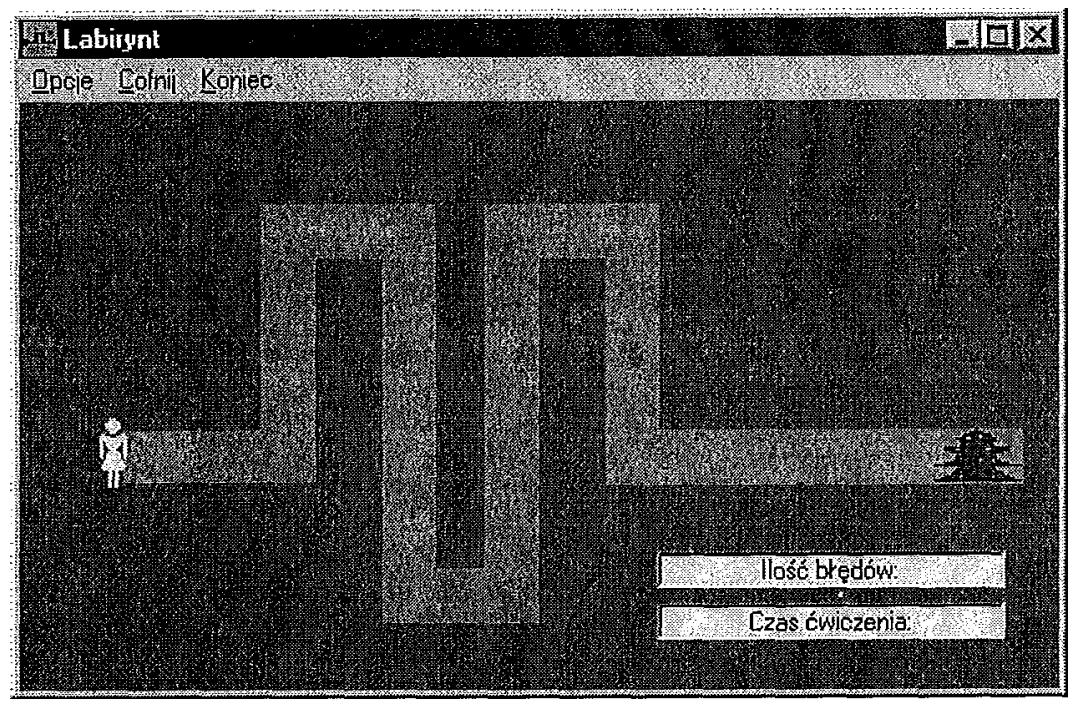

Fig. 3. The window of the subtest labyrinth (labirynt)

The task ball is illustrated in Fig. 4. The person taking the test is asked to take the ball out of the container, which is performed by driving the mouse cursor onto the left icon. Then the ball should be placed in a similar container on the right side of the screen. The idea is to move the ball as quickly as possible along the shortest possible path. The window shows the scores - the length of the path relative to the shortest possible path as well as the time of moving the ball.

The idea of the task called the circle, Fig. 5, is move the cursor within the ring displayed on the screen to complete a circle. The movement is marked by the shaded area within the ring. The task should be completed as quickly as possible and any deviation from the proper course is recorded as a mistake. To move further on, the user must go back to where the mistake was made.

Fig. 6 illustrates the task board. The person taking the test is asked to hatch the rectangular fields on the board by choosing the field and clicking the mouse. The board may be composed of 4 times 4, 4 times 8 or 8 times 8 fields. The test is completed after all the fields have been marked or after an allowed time of 120 seconds. The windows display the score, time left to complete the test and time elapsed from the start of the test. Control measurements have been made on the board with 32 fields. 


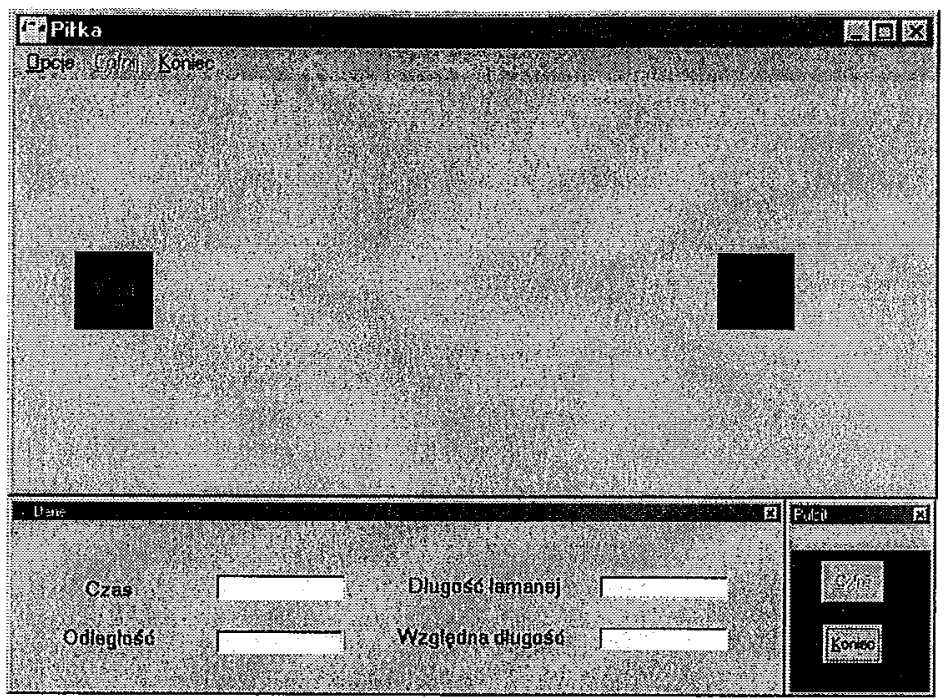

Fig. 4. The window of the subtest ball (piłka)

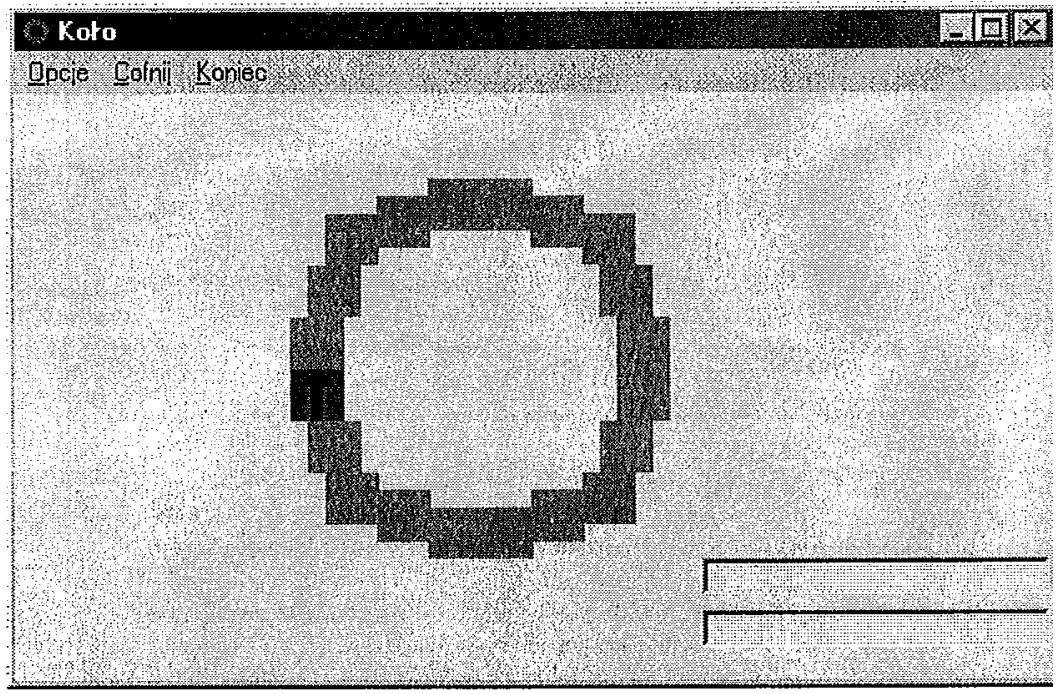

Fig. 5. The window of the subtest circle (koło)

The idea of the task centres is to mark the centres of circles displayed on the screen with the greatest possible accuracy. The window is shown in Fig. 7. The sequence of marking the centres is important, so the computer shades the already visited circles and indicates by a proper colour which circle should be visited next. The program measures the distance from the geometrical centre to the point marked. After the test, the mean distance between the point marked and the geometrical centres as well as the time of the test completion are calculated. 


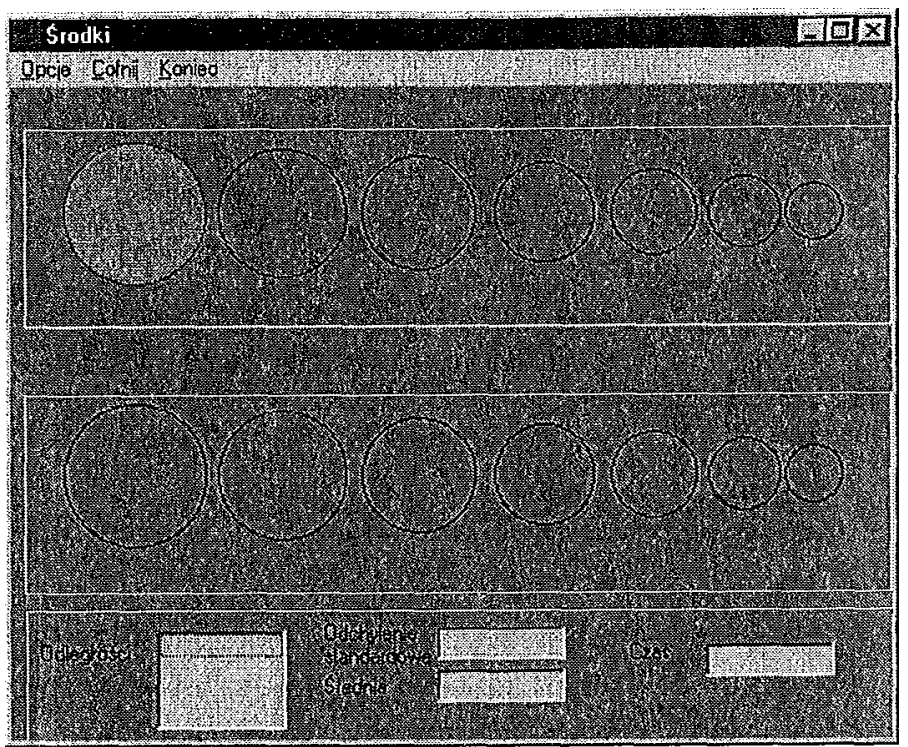

Fig. 6. The window of the subtest board (plansza)

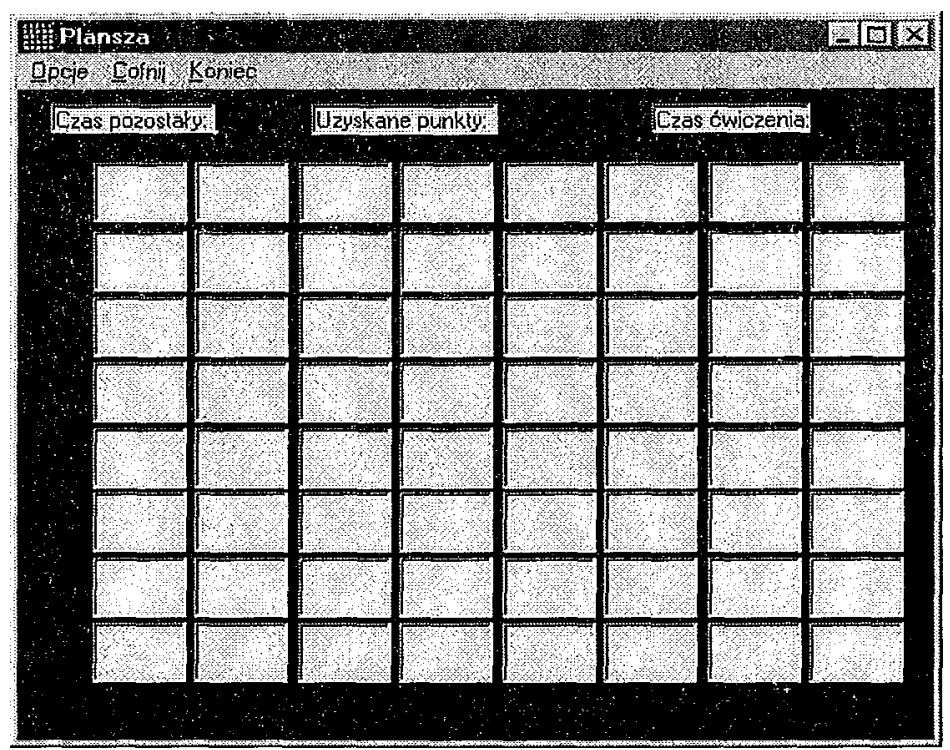

Fig. 7. The window of the subtest centres (środki)

\section{SUBJECTS TAKING THE TEST AND METHODS OF MEASUREMENTS}

The tests were performed in two elementary schools in Poznań and the subjects were randomly selected children aged from 7 to 15 . In general each child performed only 3 tasks with the two hands to eliminate the effects of getting tired and distracted attention. The number of 
children, divided into groups of healthy subjects and those with disfunctions confirmed by the Psychological and Pedagogical Institutions, is given in Table I. The population of healthy children was greater so a division was made into boys and girls, denoted by the letters $M$ and $F$, respectively. The number of children with disfunctions is given in the columns $M \& F^{*}$.

Prior to performing the test, the children were instructed about the aim and the methods of approaching the tasks, they could also check the particular applications. The tests were chosen at random to avoid the effect of selection and were performed while sitting down. Each test was performed using the left and the right hands and repeated three times with each hand. Statistical analysis was carried out for the mean results from the three attempts, so for about 3000 data. Each child was asked about contact with computer - if constant and intense the child was qualified to a group of computer-familiar children, otherwise to a group of computer non-familiar ones.

Table I. Sample size of the healthy children splitted according to age and sex ( $M$ - denotes boys and $F$ - girls) and that of children with disfunctions (denoted $M \& F^{*}$ ) irrespective of sex

\begin{tabular}{l|ccc|ccc|ccc|ccc}
\hline tests & \multicolumn{3}{|c|}{$7-8$} & \multicolumn{3}{|c|}{$9-11$} & \multicolumn{3}{c|}{$12-15$} & \multicolumn{3}{c}{ total } \\
name & $M$ & $F$ & $M \& F^{*}$ & $M$ & $F$ & $M \& F^{*}$ & $M$ & $F$ & $M \& F^{*}$ & $M$ & $F$ & $M \& F^{*}$ \\
\hline Blocks & 14 & 21 & 3 & 27 & 28 & 7 & 25 & 31 & 10 & 66 & 80 & 20 \\
Labyrinth & 14 & 23 & 3 & 26 & 29 & 7 & 24 & 27 & 11 & 64 & 79 & 21 \\
Ball & 15 & 21 & 3 & 27 & 28 & 7 & 26 & 32 & 10 & 68 & 81 & 20 \\
Circle & 19 & 12 & 3 & 26 & 25 & 6 & 27 & 26 & 10 & 72 & 63 & 19 \\
Board & 19 & 17 & 3 & 28 & 28 & 6 & 26 & 29 & 11 & 73 & 74 & 20 \\
Centres & 21 & 14 & 3 & 27 & 21 & 6 & 30 & 27 & 11 & 78 & 62 & 20 \\
\hline
\end{tabular}

\section{STATISTICAL ANALYSIS OF THE RESULTS}

The experimental data collected have been subjected to statistical processing using the program Instat. It has been established that in the aspect of normality of distribution and uniform variance, the optimum procedure for our data was to transform them to a logarithmic scale. Therefore, the times talked about in the following part of the paper should be understood as natural logarithms of the real times measured in seconds.

The first problem considered was the difference in dexterity between the dominant and nondominant hand. To analyse it, the results obtained for boys and girls in all age groups were considered jointly (in Tables II and III, columns $M \& F$ ) and subjected to the t-Student test for paired data. The results, shown in Table VI, prove a considerable statistical significance of the differences in dexterity of the dominant and non-dominant hands, usually on the level of P< 0.0001 . 
Table II. Mean values of the natural logarithms of completion time (mv) and the corresponding standard deviations (SD) for dominant hand of the healthy children. The data for boys are denoted by $M$, the data for girls - by $F$ and those for both groups $M \& F$

\begin{tabular}{lc|ccc|ccc|ccc}
\hline \multirow{2}{*}{ tests name } & & \multicolumn{3}{|c|}{$7-8$} & \multicolumn{3}{c|}{$9-11$} & \multicolumn{3}{c}{$12-15$} \\
\hline Blocks & $\mathrm{mv}$ & 2.868 & 3.254 & 3.010 & 2.479 & 2.704 & 2.585 & 2.048 & 2.392 & 2.205 \\
& $\mathrm{SD}$ & 0.444 & 0.443 & 0.476 & 0.467 & 0.417 & 0.454 & 0.406 & 0.330 & 0.408 \\
Labyrinth & $\mathrm{mv}$ & 2.977 & 3.174 & 3.057 & 2.683 & 2.742 & 2.711 & 2.283 & 2.481 & 2.376 \\
& $\mathrm{SD}$ & 0.354 & 0.414 & 0.386 & 0.477 & 0.378 & 0.430 & 0.288 & 0.317 & 0.315 \\
Ball & $\mathrm{mv}$ & 0.843 & 0.778 & 0.816 & 0.447 & 0.648 & 0.546 & 0.297 & 0.474 & 0.376 \\
& $\mathrm{SD}$ & 0.270 & 0.388 & 0.321 & 0.343 & 0.257 & 0.318 & 0.403 & 0.354 & 0.388 \\
Circle & $\mathrm{mv}$ & 2.478 & 2.582 & 2.542 & 2.228 & 2.421 & 2.326 & 1.835 & 2.016 & 1.927 \\
& $\mathrm{SD}$ & 0.334 & 0.483 & 0.428 & 0.338 & 0.421 & 0.391 & 0.326 & 0.399 & 0.373 \\
Board & $\mathrm{mv}$ & 4.006 & 4.106 & 4.059 & 3.680 & 3.747 & 3.714 & 3.191 & 3.393 & 3.286 \\
& $\mathrm{SD}$ & 0.511 & 0.389 & 0.447 & 0.452 & 0.440 & 0.443 & 0.383 & 0.353 & 0.380 \\
Centres & $\mathrm{mv}$ & 3.276 & 3.425 & 3.365 & 3.089 & 3.022 & 3.051 & 2.591 & 2.839 & 2.713 \\
& $\mathrm{SD}$ & 0.400 & 0.396 & 0.399 & 0.370 & 0.488 & 0.437 & 0.405 & 0.405 & 0.420 \\
\hline
\end{tabular}

Table III. Mean values of the natural logarithms of completion time (mv) and the corresponding standard deviations (SD) for non-dominant hand of healthy children

\begin{tabular}{ll|ccccccccc}
\hline tests name & \multicolumn{3}{|c}{$7-8$} & \multicolumn{3}{c}{$9-11$} \\
& & $M$ & $F$ & $M \& F$ & $M$ & $F$ & $M \& F$ & $M$ & $F$ & $M \& F$ \\
\hline Blocks & $\mathrm{mv}$ & 3.080 & 3.525 & 3.278 & 2.735 & 2.931 & 2.833 & 2.471 & 2.600 & 2.529 \\
& $\mathrm{SD}$ & 0.293 & 0.526 & 0.464 & 0.442 & 0.389 & 0.424 & 0.345 & 0.304 & 0.331 \\
Labyrinth & $\mathrm{mv}$ & 3.501 & 3.695 & 3.575 & 3.294 & 3.274 & 3.285 & 2.887 & 2.905 & 2.895 \\
& $\mathrm{SD}$ & 0.435 & 0.481 & 0.456 & 0.473 & 0.351 & 0.415 & 0.341 & 0.344 & 0.339 \\
Ball & $\mathrm{mv}$ & 1.042 & 1.183 & 1.101 & 0.827 & 0.873 & 0.850 & 0.463 & 0.665 & 0.559 \\
& $\mathrm{SD}$ & 0.307 & 0.313 & 0.313 & 0.315 & 0.382 & 0.347 & 0.458 & 0.339 & 0.415 \\
Circle & $\mathrm{mv}$ & 2.733 & 2.916 & 2.845 & 2.372 & 2.569 & 2.473 & 2.104 & 2.351 & 2.227 \\
& $\mathrm{SD}$ & 0.483 & 0.501 & 0.494 & 0.239 & 0.457 & 0.376 & 0.333 & 0.445 & 0.40 \\
Board & $\mathrm{mv}$ & 4.330 & 4.357 & 4.344 & 3.895 & 4.090 & 3.993 & 3.535 & 3.772 & 3.649 \\
& $\mathrm{SD}$ & 0.266 & 0.255 & 0.257 & 0.377 & 0.293 & 0.349 & 0.246 & 0.281 & 0.287 \\
Centres & $\mathrm{mv}$ & 3.411 & 3.588 & 3.515 & 3.281 & 3.190 & 3.233 & 2.841 & 3.104 & 2.973 \\
& $\mathrm{SD}$ & 0.356 & 0.384 & 0.378 & 0.319 & 0.301 & 0.310 & 0.400 & 0.383 & 0.410 \\
\hline
\end{tabular}

The descriptive statistics of the results of measurements was performed using a specially prepared computer program. The analysis was performed for three age groups: group I of 7 and 8 year olds, group II including children of 9 to 11 years of age and elder children were in group III. The mean times of tests completion and other characteristics as well as standard deviations were calculated. The times of task completions by the children grouped for each hand, sex, age groups, computer-familiar or non-familiar categories are collected in Tables from II to V. 
Table IV. Mean values of the natural logarithms of completion time (mv) and the corresponding standard deviations (SD) for healthy children in the given age groups splitted into subgroups $C$ (familiar with computer) and $N$ (non-familiar with computer). Data for the dominant hand

\begin{tabular}{l|cc|cc|cc|cc|cc|cc}
\hline \multirow{2}{*}{ tests name } & \multicolumn{2}{|c|}{$C$} & \multicolumn{2}{|c|}{$N$} & \multicolumn{2}{c|}{$C$} & \multicolumn{2}{c|}{$N$} & \multicolumn{2}{c}{ C } & \multicolumn{2}{c}{$N$} \\
& $\mathrm{mv}$ & $\mathrm{SD}$ & $\mathrm{mv}$ & $\mathrm{SD}$ & $\mathrm{mv}$ & $\mathrm{SD}$ & $\mathrm{mv}$ & $\mathrm{SD}$ & $\mathrm{mv}$ & $\mathrm{SD}$ & $\mathrm{mv}$ & $\mathrm{SD}$ \\
\hline Blocks & 2.84 & 0.52 & 3.15 & 0.39 & 2.30 & 0.37 & 2.88 & 0.31 & 1.93 & 0.22 & 2.48 & 0.36 \\
Labyrinth & 2.82 & 0.28 & 3.25 & 0.07 & 2.51 & 0.39 & 2.93 & 0.35 & 2.23 & 0.04 & 2.55 & 0.06 \\
Ball & 0.75 & 0.41 & 0.87 & 0.17 & 0.41 & 0.34 & 0.68 & 0.22 & 0.15 & 0.31 & 0.62 & 0.30 \\
Circle & 2.35 & 0.42 & 2.69 & 0.38 & 2.10 & 0.30 & 2.52 & 0.35 & 1.72 & 0.28 & 2.13 & 0.33 \\
Board & 3.75 & 0.41 & 4.30 & 0.31 & 3.41 & 0.37 & 3.97 & 0.32 & 3.02 & 0.25 & 3.55 & 0.29 \\
Centres & 3.21 & 0.41 & 3.49 & 0.34 & 2.81 & 0.42 & 3.26 & 0.32 & 2.49 & 0.31 & 2.91 & 0.40 \\
\hline
\end{tabular}

Table V. Mean values of the natural logarithms of completion time and the corresponding standard deviations (SD) for the dominant (RD) and non-dominant hand (RND) of children with disfunctions.

The first column in a given subgroup shows the mean values whereas the second column - SD

\begin{tabular}{l|cl|cc|cc|cc|cc|cc}
\hline tests name & \multicolumn{4}{|c|}{$7-8$} & \multicolumn{4}{c|}{$9-11$} & \multicolumn{3}{c}{$12-15$} \\
& \multicolumn{2}{|c}{ RD } & \multicolumn{2}{c|}{ RND } & \multicolumn{2}{c}{ RD } & \multicolumn{2}{c}{ RND } & \multicolumn{2}{c}{ RD } & RND \\
\hline Blocks & 4.093 & 0.230 & 4.125 & 0.715 & 3.128 & 0.567 & 3.260 & 0.615 & 2.491 & 0.339 & 2.717 & 0.343 \\
Labyrinth & 3.457 & 0.490 & 4.087 & 0.549 & 3.482 & 0.714 & 3.967 & 0.777 & 2.843 & 0.569 & 3.036 & 0.350 \\
Ball & 1.060 & 0.122 & 1.205 & 0.107 & 0.952 & 0.453 & 1.418 & 0.593 & 0.568 & 0.303 & 0.801 & 0.351 \\
Circle & 2.607 & 0.652 & 2.633 & 0.633 & 2.501 & 0.517 & 2.491 & 0.397 & 2.066 & 0.456 & 2.354 & 0.482 \\
Board & 4.317 & 0.551 & 4.667 & 0.205 & 4.028 & 0.443 & 4.153 & 0.532 & 3.371 & 0.371 & 3.548 & 0.272 \\
Centres & 3.899 & 0.143 & 3.705 & 0.518 & 2.995 & 0.400 & 3.275 & 0.372 & 2.992 & 0.418 & 3.248 & 0.446 \\
\hline
\end{tabular}

Table VI. The $\mathrm{P}$ values found from the $t$-Student tests on the statistical significance of the differences between mean values of completion time for the dominant and non-dominant hands of healthy children

\begin{tabular}{l|ccc}
\hline tests name & $7-8$ & $9-11$ & $12-15$ \\
\hline Blocks & $P<0.0001$ & $P<0.0001$ & $P<0.0001$ \\
Labyrinth & $P<0.0001$ & $P<0.0001$ & $P<0.0001$ \\
Ball & $P<0.0001$ & $P<0.0001$ & 0.0006 \\
Circle & $P<0.0001$ & 0.0023 & $P<0.0001$ \\
Board & 0.0009 & $P<0.0001$ & $P<0.0001$ \\
Circles & 0.0093 & 0.0125 & 0.0002 \\
\hline
\end{tabular}

A similar analysis was made to check if the results differ significantly for boys and girls. In groups of younger children, the $t$-Student test analysis did not reveal significant differences, although with some exceptions specified in Table VII (see the columns $M v s F$ ). In the eldest group, the differences for boys and girls were statistically significant in 4 cases.

Another problem considered was the difference between computer-familiar children and others from the population of healthy children. The results of $t$-Student tests for the dominant 
hand revealed such statistically significant differences with some exceptions in the group of the youngest children (Table VII, columns $C$ vs N).

Table VII. The $P$ values found from the t-Student tests on the statistical significance of differences between data for healthy boys and girls $(M \vee s F)$ and those for children familiar and non-familiar with computer $(C v s N)$

\begin{tabular}{l|llllll}
\hline \multirow{2}{*}{ tests name } & \multicolumn{2}{|c}{$7-8$} & \multicolumn{2}{c}{$9-11$} & \multicolumn{2}{c}{$12-15$} \\
& $M v s F$ & $C v s N$ & $M v s F$ & $C v s N$ & $M v s F$ & $C v s N$ \\
\hline Blocks & 0.0072 & 0.0546 & 0.0655 & 0.0001 & 0.0012 & 0.0001 \\
Labyrinth & 0.3268 & 0.0003 & 0.6177 & 0.0001 & 0.0238 & 0.0001 \\
Ball & 0.5803 & 0.2410 & 0.0175 & 0.0013 & 0.0817 & 0.0001 \\
Circle & 0.4225 & 0.0420 & 0.0784 & 0.0001 & 0.0774 & 0.0001 \\
Board & 0.5114 & 0.0001 & 0.5778 & 0.0001 & 0.0478 & 0.0001 \\
Centres & 0.2865 & 0.0361 & 0.5881 & 0.0002 & 0.0305 & 0.0001 \\
\hline
\end{tabular}

In the next step we wanted to check the differences in hand dexterity between different age groups and possibly establish clinical standards. The variance analysis of the results for the dominant (Table VIII - RD) as well as non-dominant (Table VIII - RDN) hands proved the statistically significant differences between the average results in all age groups. Therefore, the data given in Tables II and III should be treated as standard values for healthy children, and the systematic decrease in the time of tests completion with age should be considered a statistically important feature describing increasing dexterity of hands with age. The graphical representation of the relevant data listed in Tables II and III is plotted in Figs. 8 and 9 for the dominant and non-dominant hand, respectively. Strong correlations of the results with the children age are illustrated in Fig. 10 which illustrates the mean values of the real time completion of all the tasks for the dominant hand of the healthy children versus their age. The graphs refer to: $A$ - blocks, $B$ - circle, C - ball, $D$ - labyrinth, $E$ - board, $F$ - centres, respectively. The values $r$ displayed in the corners stand for the Pearson correlation coefficients. The significance of the correlation has not been proven, however.

Table VIII. The results of ANOVA analysis of differences between the given age groups of healthy children

\begin{tabular}{l|ll|ll}
\hline \multirow{2}{*}{ tests name } & \multicolumn{2}{|c|}{$7-8 v s 9-11$} & \multicolumn{2}{|c}{$9-11$ vs $12-15$} \\
& \multicolumn{1}{|c|}{ RD } & RND & \multicolumn{1}{c}{ RD } & \multicolumn{1}{c}{ RND } \\
\hline Blocks & $P<0.001$ & $P<0.001$ & $P<0.001$ & $P<0.001$ \\
Labyrinth & $P<0.001$ & $\mathrm{P}<0.01$ & $P<0.001$ & $P<0.001$ \\
Ball & $\mathrm{P}<0.01$ & $\mathrm{P}<0.01$ & $P<0.05$ & $P<0.001$ \\
Ball & $P<0.05$ & $P<0.001$ & $P<0.001$ & $P<0.01$ \\
Board & $P<0.001$ & $P<0.001$ & $P<0.001$ & $P<0.001$ \\
Centres & $P<0.01$ & $P<0.01$ & $P<0.01$ & $P<0.01$ \\
\hline
\end{tabular}


Table IX. The $P$ values showing the level of significance of differences between the corresponding mean values for the healthy children and those with disfunctions

\begin{tabular}{l|cc|cc|cc}
\hline \multirow{2}{*}{ test name } & \multicolumn{2}{|c|}{$7-8$} & \multicolumn{2}{c|}{$9-11$} & \multicolumn{2}{c}{$12-15$} \\
& RD & RND & RD & RND & RD & RND \\
\hline Blocks & 0.0009 & 0.0446 & 0.0055 & 0.0214 & 0.0395 & 0.1039 \\
Labyrinth & 0.2107 & 0.1164 & 0.0106 & 0.0630 & 0.0117 & 0.2392 \\
Ball & 0.1023 & 0.0009 & 0.0036 & 0.0196 & 0.1447 & 0.2630 \\
Circle & 0.9091 & 0.0984 & 0.2908 & 0.9031 & 0.3014 & 0.3844 \\
Board & 0.3167 & 0.0512 & 0.1039 & 0.3141 & 0.5018 & 0.3298 \\
Centres & 0.4015 & 0.4695 & 0.7669 & 0.7607 & 0.0494 & 0.0595 \\
\hline
\end{tabular}

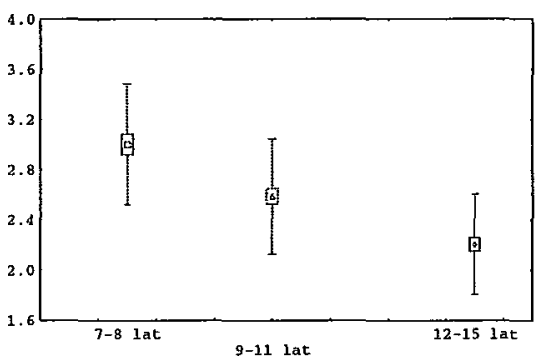

A-blocks

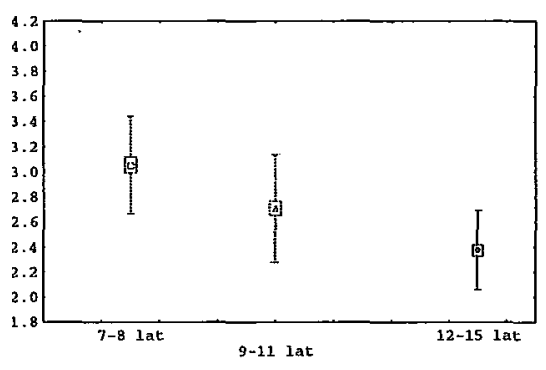

C-ball

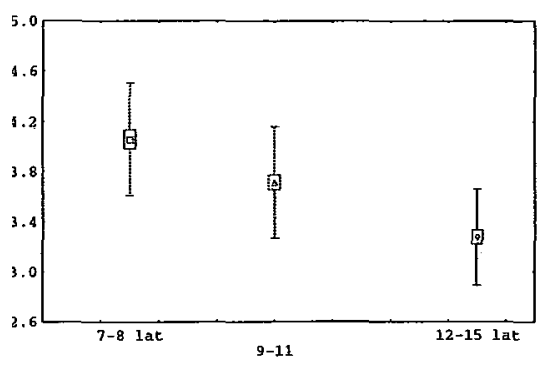

$\mathrm{E}$ - board

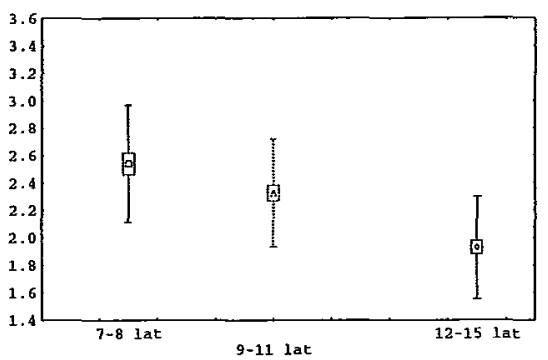

B-circle
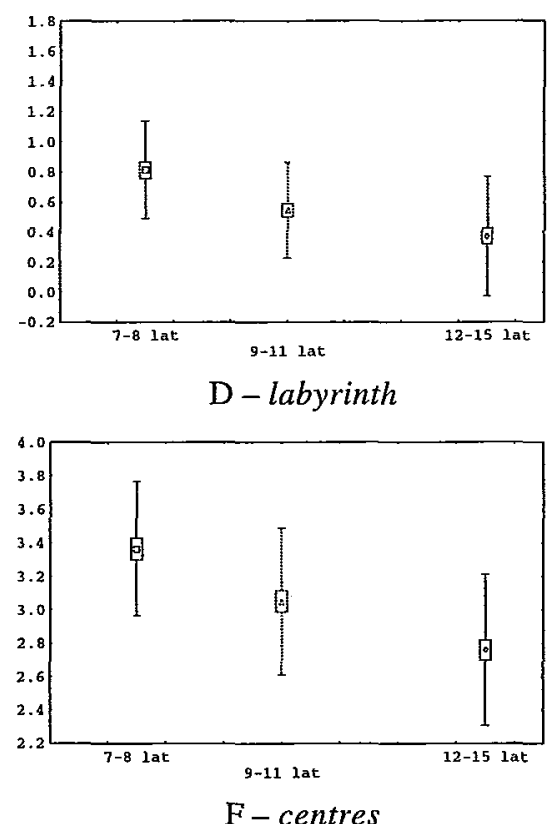

Fig. 8. The standardised data of the logarithms of completion time for the dominant hand in three age groups. The rectangles denote the SEM values and the segments of a line - the corresponding SD values 
Relatively few results obtained for the children with disfunctions confirmed by Psychological and Pedagogical Institutions, have also been analysed, using non-parametric test. Although the mean values differ in age groups (Table V), they proved to be statistically insignificant. A comparison of the results obtained in groups II and III with the corresponding values for healthy children (Table IX) reveals statistically significant differences in half of the cases.
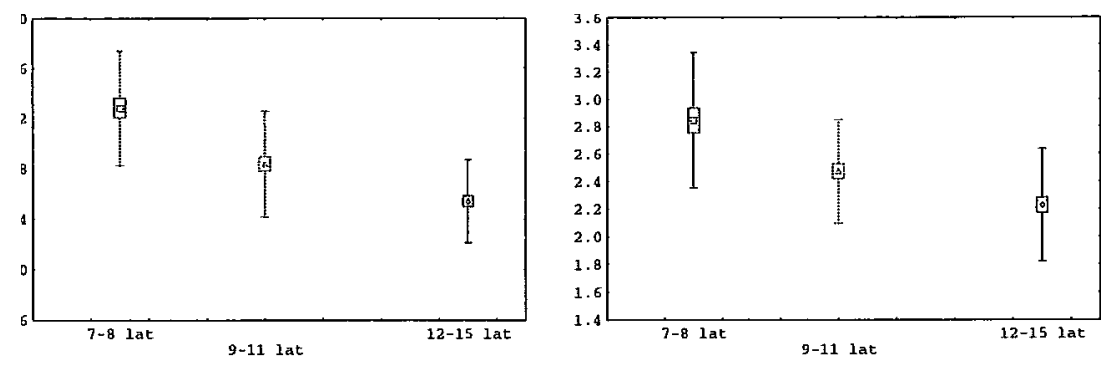

A - blocks
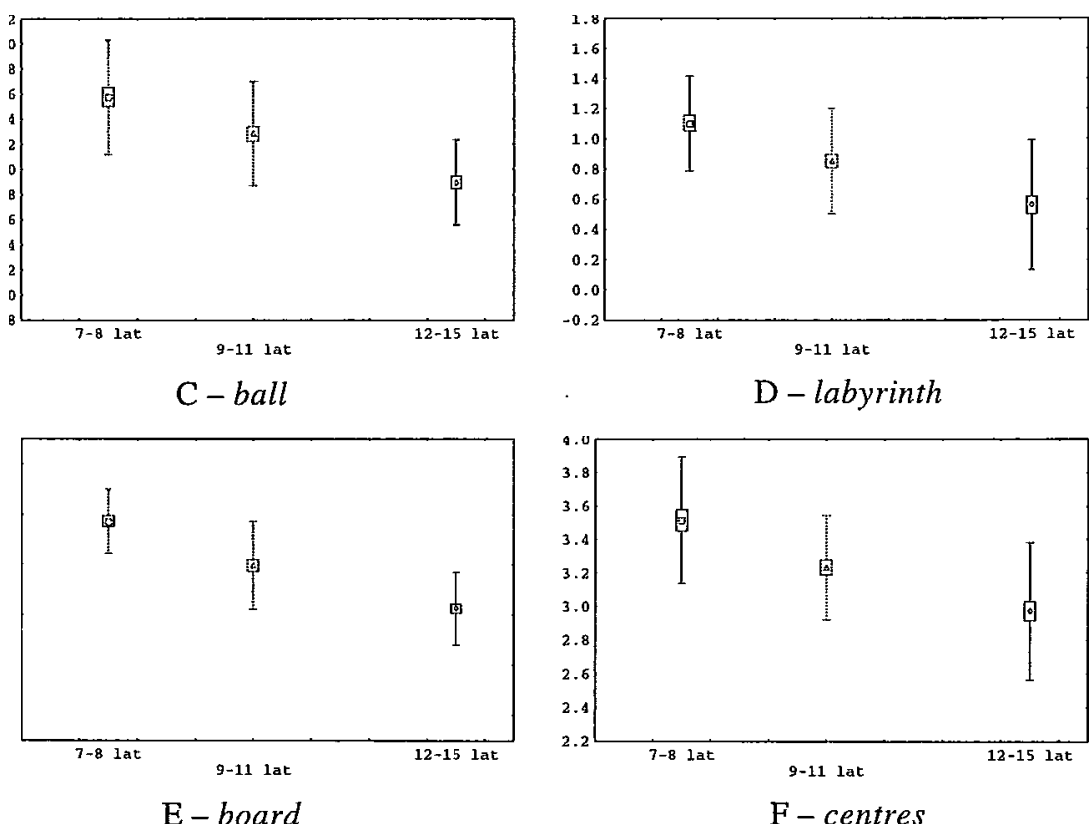

Fig. 9. The standardised data of the logarithms of completion time for the non-dominant hand in three age groups. The rectangles denote the SEM values and the segments of a line - the corresponding SD values

It should be mentioned that the disfunctions following from a minimal brain injury, leading to affected eye - hand coordination, psycho-motoric hyperactivity and other symptoms, did not prevent the children from normal functioning and progress among their peers. The results do not exclude that the application of our computer tests on the population of children with disfunctions of the upper limbs will prove useful for assessment of hand dexterity and will show a sufficient correlation with the results describing the functional status of the hand. 

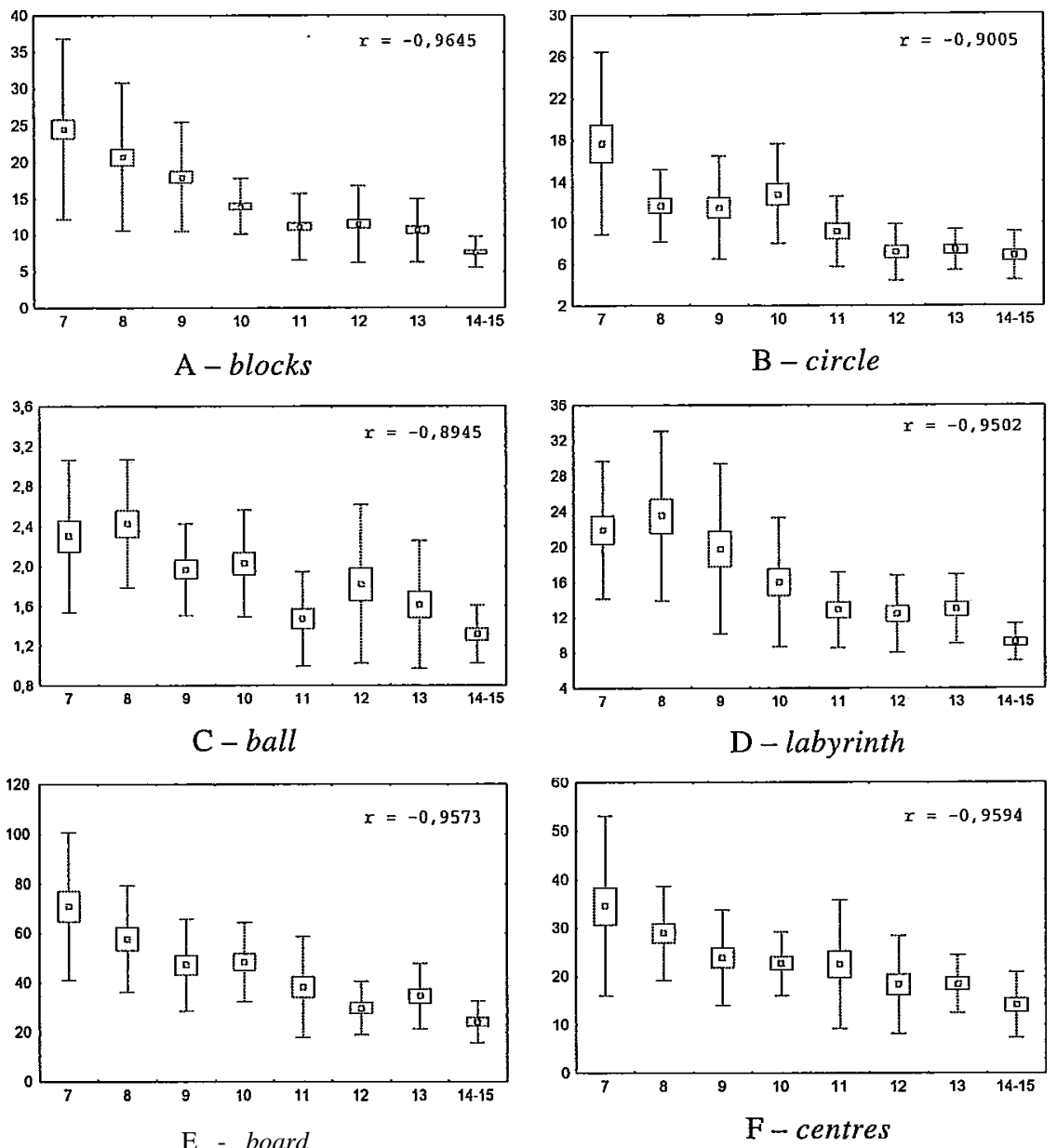

Fig. 10. The real values of the task completion time (in seconds) for the dominant hand of healthy children versus the age. The corresponding Pearson coefficients are given in the corners

\section{CONCLUSIONS}

1) New computer tests checking the dexterity of both hands have been developed in the Visual Basic language on the Windows platforms.

2) Standard values of natural logarithms of time (in seconds) required to complete particular tests for children from particular age groups have been established.

3) Statistical significance of the differences in mean logarithms of times needed to complete all tests for selected groups of healthy children (healthy in the sense of no disfunctions confirmed by Psychological and Pedagogical Institutions) has been proved. 


\section{Acknowledgements}

We thank Prof. J. Moczko and Mr. L. Dębski for helpful discussions, and Prof. J. Rogiers for the critical reading of the manuscript.

\section{References}

[1] W. Cytowicz-Karpiłowska and A. Seyfried, Postępy Rehabil. IX, 37 ( 1995).

[2] M. Kellor, J. Frost, N. Silberberg, I. Iversen, R. Cummings, Am. J. Occup. Ther. 25, 77 (1971).

[3] K. G. Thorngren and C. O. Werner, Acta Orthop. Scand. 50, 255 (1979).

[4] V. Mathiowetz, K. Weber, G. Volland, N. Kashman, J. Hand Surg. 9A, 222 (1984).

[5] R. T. Schmidt and J. V. Toews, Arch. Phys. Med. Rehabil. 51, 321 (1970).

[6] J. C. Pryce, J. Biomechanics, 13, 505 (1980).

[7] V. Mathiowetz, N. Kashman, G. Volland, K. Weber, M. Dowe, S. Rogers, Arch. Phys. Med. Rehabil. 66, 69 (1985).

[8] R. Izquierdo-Avino, R. S. Jones, B. Jansen, E. Schwemm, J. Orthop. Reumatol. 8, 203 (1995).

[9] B. M. Koch and R. L Simenson, Arch. Phys. Med. Rehabil. 73, 241 (1992).

[10] T. Jarus and R. Poremba, Am. J. Occup. Ther. 47, 439 (1993).

[11] R. H. Jebsen, N. Taylor, R. B. Trieschman, M. J. Trotter, L. A. Howard, Arch. Phys. Med. Rehabil., 50,311 (1969).

[12] T. P. Vliet Vlieland, T. P. van der Wijk, I. M. Jolie, A. H. Zwinderman, J. M. Hazes, J. Reumatol., 23, 835 (1996).

[13] J. H. Carr, R. B Shepherd, L. Nordholm, D. Lynne, Phys. Ther. 65, 175 (1985).

[14] D. T. Wade, R. Langton-Hewer, V. A. Wood, C. E. Skilbeck, H. M. Ismail, J. Neurol. Neurosurg. Psychiatry 46, 521 (1983).

[15] V. M. Parker, D. T. Wade, R. L. Hewer, Int. Rehabil. Med. 8, 69 (1986).

[16] V. Mathiowetz, G. Volland, N. Kashman, K. Weber, Am. J. Occup. Ther. 39, 386 (1985).

[17] J. Desrosiers, G. Bravo, R. Hebert, E. Dutil, L. Mercier, Arch. Phys. Med. Rehabil. 75, 751 (1994).

[18] Cz. Piskorz and E. Klimek-Piskorz, Post. Rehabil. IX, 31 (1995).

[19] C. Stein and E. J. Yerxa, Am. J. Occup. Ther. 44, 499 (1990).

[20] J. Dylewicz, A. Mołczanow, G. Radecka, Materiały Sesji Naukowej PAN, Poznań, 84 (1983).

[21] S. van Langveld, P. van't Pad Bosch, J. Bakker, S. Terwindt, M. Fraussen, P. van Riel,

J. Hand Ther. 9, 27(1996).

[22] D. Reddihough, T. Bach, G. Burgess, L. Oke, I. Hudson, Develop. Med. Child Neurol. 33, 578 (1991).

[23] N. J. O'Dwyer, L. Ada, P. D. Neilson, Brain, 119, 1737 (1996).

[24] S. D. Mc Phee, Am. J. Occup. Ther. 41, 158 (1987). 